\title{
Review
}

Mari Carmen Bernal-Soriano*, Blanca Lumbreras, Ildefonso Hernández-Aguado, María Pastor-Valero, Maite López-Garrigos and Lucy A. Parker

\section{Untangling the association between prostate- specific antigen and diabetes: a systematic review and meta-analysis}

https://doi.org/10.1515/cclm-2020-0145

Received February 12, 2020; accepted May 23, 2020; published online July 20,2020

\section{Abstract}

Objectives: Several studies have shown an inverse association between diabetes mellitus and prostate cancer (PCa). Some researchers suggest that this relationship is due to reduced PCa detection in diabetics due to lower prostate-specific antigen (PSA) levels compared to nondiabetics. Our objective is to analyze the impact of diabetes on PSA in asymptomatic men without known prostate pathology and without prior prostate intervention.

Methods: We searched Medline (via PubMed), Embase and Scopus. We included studies that reported the relationship between serum PSA levels and diabetes or diabetes treatment in asymptomatic adult men without known prostate pathology, and without prior prostate intervention. Pooled mean differences were compared between diabetics and non-diabetics.

Results: Of 2,392 screened abstracts, thirteen studies met the inclusion criteria and $8(62 \%)$ reported appropriate measures that could be included in a meta-analysis. Eleven (85\%) examined the influence of diabetes on PSA levels and $8(62 \%)$ evaluated the influence of diabetes treatments on PSA levels. Overall diabetics had a significantly lower PSA level compared to non-diabetics (mean difference: $-0.07 \mathrm{ng} / \mathrm{mL}$; $95 \% \mathrm{CI}-0.10,-0.04)$.

*Corresponding author: Mari Carmen Bernal-Soriano, Department of Public Health, University Miguel Hernández, Crta. Nacional, N-332, s/n, Sant Joan, 03550, Alicante, Spain; CIBER de Epidemiología y Salud Pública (CIBERESP), Madrid, Spain,

E-mail: maria.bernals@umh.es, Phone: +34 965919516. Blanca Lumbreras, Ildefonso Hernández-Aguado, María PastorValero and Lucy A. Parker: Department of Public Health, University Miguel Hernández, Crta. Nacional, N-332, s/n, Sant Joan, Alicante, Spain; CIBER de Epidemiología y Salud Pública (CIBERESP), Madrid, Spain

Maite López-Garrigos: Clinical Laboratory Department, University Hospital of San Juan de Alicante, Alicante, Spain
Conclusions: Diabetes and related factors (such as disease duration, severity and treatment) were significantly associated with lower PSA levels among asymptomatic men, yet differences were small and are unlikely to influence PCa detection in a screening setting.

Keywords: diabetes mellitus; prostate cancer screening; prostate-specific antigen.

\section{Introduction}

Prostate-specific antigen (PSA) testing, followed by biopsy when PSA level is over $3-4 \mathrm{ng} / \mathrm{mL}$ is a screening method accepted by both the American Urology Association (AUA) [1] and the European Association of Urology (EAU) [2]. The screened population should be well-informed patients in good health, with a life expectancy of at least 10-15 years. They should include high-risk groups such as AfroAmericans or men with a family history of prostate cancer [3]. The U.S. Preventive Services Task Force (USPSTF) updated its recommendations in 2017, suggesting that after doctors have explained the benefits and risks of PSA screening to the patient, they should jointly take the decision of whether or not to carry out the test [4].

However, the PSA test presents several limitations lack of a universally accepted threshold value [5, 6]. Regarding validity, factors such as age, acute prostatitis, ejaculation, catheterization and certain comorbidities and medications influence PSA levels [7]. The relation of PSA, prostate cancer and diabetes deserves particular attention.

Several studies have evaluated the relationship between diabetes mellitus (DM) and PCa. A meta-analysis provided strong evidence supporting an inverse association between the two conditions [8]. There are several hypotheses underlying this inverse association. Some researchers have suggested that long-term diabetes could reduce the testosterone levels and others have indicated that this reduction is associated with cancer-related growth factors among diabetics [8]. Furthermore, diabetes-induced vascular damage in the 
prostate has been suggested to have protective effect by limiting tumor growth [9]. On the other hand, some researchers suggest the inverse relationship is due to reduced detection of PCa among diabetics due to lower PSA levels compared with non-diabetics, again attributed to lower levels of testosterone [10]. In this case, diabetics would be less likely to have a positive PSA result than non-diabetics and consequently, they would be less likely to have a biopsy and subsequent early diagnosis of PCa [9]. As a result, diabetics may be more likely to develop high-grade disease before diagnosis compared to non-diabetics.

The most commonly used PSA threshold (3-4 ng/mL) for determining the need of a biopsy does not consider differences between diabetics and non-diabetics. The available recommendations do not include different strategies to be adopted after a PSA result according to the presence of DM. Hence, if the usual threshold of PSA $(3-4 \mathrm{ng} / \mathrm{mL})$ is used for both diabetics and non-diabetics, smaller tumors could be missed in diabetics.

We hypothesize that the lower range of PSA values identified in diabetics compared to non-diabetics may justify a different cut-off point when considering a positive PSA result in this population. The aim of this systematic review and meta-analysis is to analyze the different PSA levels between diabetics and non-diabetics in asymptomatic men without known prostate pathology and without prior prostate intervention.

\section{Methods}

The review was registered on March 1, 2017 (PROSPERO 2017 CRD42017058661). Available from: http://www.crd.york.ac. uk/PROSPERO/display_record.php?ID=CRD42017058661.

The review is reported in accordance with "The Preferred Reporting Items for Systematic Reviews and Meta-Analyses: The PRISMA Statement” [11].

\section{Search strategy}

In July 2018 (10 July 2018), we searched the Medline (via PubMed), Embase and Scopus using free text and MESH terms with no time limit. The search terms were as follows: "Diabetes", "Diabetes Mellitus" [Mesh], "Prostate-Specific Antigen" [Mesh], "Prostate-Specific Antigen" and Medline search also contained the terms: "Obesity", "Body Mass Index", "Body Mass Index" [Mesh], "BMI", "Obesity" [Mesh]. A second search was conducted in March 2020 to update the list of references using the same search strategy. We added a limit (publication date) in order to recover manuscripts published from July 2018 until December 2019.

\section{Inclusion criteria}

We defined the inclusion criteria based on a specific population (P), intervention (I), comparator (C) and outcome (O), as recommended by PRISMA. We included original research studies that reported the differences in serum PSA levels (O) between diabetics (P) and nondiabetics (C) and/or patients undergoing diabetes medication compared (P) to no treatment (C) in asymptomatic adult men (aged over 18 years) without prostate pathology, and without any prior prostate intervention (P). When the study included men who had been diagnosed with PCa or men with prostate symptoms, only results relating to undiagnosed or asymptomatic men were considered for this review. We selected publications in English, French or Spanish.

\section{Systematic review process}

Two independent researchers examined the titles and abstracts to preselect articles for full text review. We also carried out full text review in duplicate. Discrepancies were resolved by discussion and arbitration with a third researcher. The PRISMA flowchart illustrating the search and selection process is provided in Figure 1.

\section{Data extraction}

Two authors, using a data extraction form defined a priori, extracted data independently. We extracted the following data from each study: objective, study design, selection criteria (inclusion/exclusion criteria), population source and setting, age and race of the study population, sample size (n), other characteristics of the study population, follow-up (yes/no and time), exposure: BMI, diabetes (and treatments with drug doses), additional covariates, whether additional covariates were used to adjust and/or stratify PSA results, PSA value (mean) for each subgroup, laboratory technology used to determine PSA, main results of the study, study limitations, conclusion of the study, and any comments made by the authors regarding the implications of the results for screening/diagnosis. Disagreement was solved by discussion and arbitration with a third researcher.

Although all studies explored the association between PSA and diabetes, we further classified the studies into the following categories: (a) patients with diagnosis of diabetes; (b) patients undergoing treatment for diabetes, and (c) patients with some clinical factors related to diabetes (such as insulin resistance). 


\section{Quality assessment}

We carried out a formal analysis of study quality and risk of bias using the relevant dimensions from existing risk of bias tools [12-14]. Specifically, we evaluated the likeliness of selection bias as described in the Quality Assessment Tool for Quantitative Studies [14], we explored the potential for bias in the ascertainment of the exposure as per the Newcastle-Ottawa Scale (NOS) for assessing the quality of non-randomised studies in meta-analyses [12] and finally, in line with guidance from the Cochrane Risk of Bias Assessment Tool: for Non-Randomized Studies of Interventions [13], we identified that age and BMI would be important potential confounders in the context of the studies, and considered the relevant questions to assess baseline confounding. We deemed this strategy most appropriate for evaluating study quality because no single guideline covered the mix of designs and methodologies that were included, and the full guidelines included numerous items that were not applicable to studies given their cross sectional design. Each quality item was assessed in duplicate and blinded. Disagreement was solved by discussion and arbitration with a third researcher.

\section{Statistical analysis}

In addition to a systematic review of all the articles included, we carried out a meta-analysis with all those studies that included PSA levels according to the presence or absence of diabetes. We used the summarized results that were published in each study rather than requesting the original data.

In studies that reported medians and ranges, we estimated the means and standard deviations [15, 16]. Furthermore, for one study that reported PSA results in diabetics with and without treatment, both subgroups were

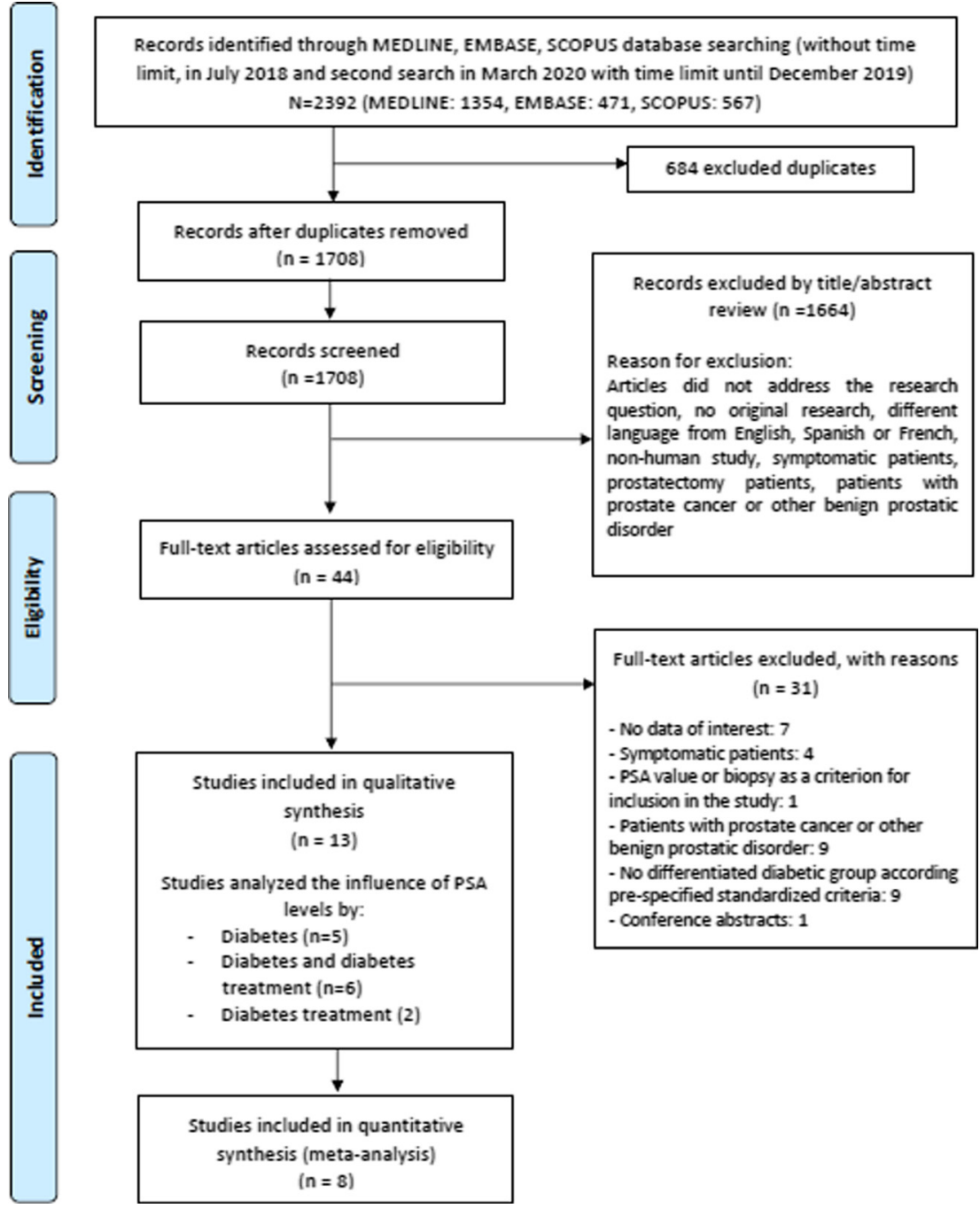

Figure 1: Flow diagram detailing the search and selection process. 
combined to have a larger single sample according to the Cochrane Handbook [17].

Pooled mean differences were compared between diabetics and non-diabetics with adjustments for age or BMI whenever possible. A subgroup analysis compared participants $<60$ and $\geq 60$ years. Heterogeneity among the studies was assessed by the Cochran $Q$ and the $\mathrm{I}^{2}$ statistic. If either the Q statistic value was $<0.1$ or $\mathrm{I}^{2}$ was $>50 \%$, a random effects model of analysis was used. The withinstudy differences between exposed and unexposed groups were assessed as potential heterogeneity sources of results by using sensitivity and meta-regression analyses. The following covariables were considered: age (no differences, differences or not informed between a diabetic and non-diabetic group); less than $20 \%$ of subjects in the diabetic group; the design of the study (cohort, cross-sectional, case-control design) and study quality (strong, moderate, weak). p-values $<0.05$ were considered to be statistically significant. Publication bias was evaluated using the Begg method. We used Stata, version 15 (StataCorp LP; College Station, TX) to perform all analyses.

\section{Results}

\section{Search results}

A flow diagram of study selection is shown in Figure 1. Out of 2,392 studies evaluated, 13 were included in the systematic review, and 8 of them (62\%) reported measures that could be included in a meta-analysis (such as mean of PSA values by subgroups of diabetics and nondiabetics) and three articles allowed an age-stratified analysis (participants $<60$ and $\geq 60$ years). The characteristics of the included studies are summarized in Table 1 (see Supplementary Table S1 for additional details such as population source, inclusion and exclusion criteria). Studies were published between 2006 and 2019. Among all the studies included, 11 (85\%) studies examined the influence of diabetes on PSA levels and $8(62 \%)$ evaluated the influence of diabetes treatments on PSA levels.

\section{Influence of diabetes on PSA levels}

Out of the 11 studies included [18-28], 8 (73\%) were crosssectional; 2 (18\%) were cohort studies and 1 (9\%) used a case-control design. They included men aged at least 35 years old. Three studies found significant differences in
PSA levels between diabetics and non-diabetics in the crude analysis $[19,23,27]$ and three studies that did not report the crude PSA levels, found significant differences in PSA levels between diabetics and non-diabetics after adjustment by confounding factors (age, BMI, race) [24, 26, 28]. Two studies that did not find significant differences in the crude analysis [18, 22], showed association of diabetes and PSA in the adjusted analysis. In summary, eight studies showed lower PSA levels among diabetes and two studies did not find differences (see Supplementary Table S2 for additional details).

There were particularities that are worth mentioning. One of the studies [19] showed a different pattern of association between PSA level and age among diabetics and non-diabetics. In non-diabetic populations, PSA level increased significantly with age (correlation $r=0.463$; $\mathrm{p}<0.0001$ ), but in diabetics, it increased with age until age of 60 years and then it decreased, resulting in no significant association between serum PSA and age in this group (correlation $\mathrm{r}=0.141, \mathrm{p}=0.242$ ). Furthermore, PSA level in diabetics were significantly lower for higher BMI categories $(\mathrm{p}=0.037)$ [19].

Several studies obtained differences by adjusting $[22-24,26]$ or stratifying for age $[18,22,25]$. One of these studies [23] showed little effect on this association when BMI was also included in the model. In racestratified analysis they observed significant differences in BMI- and age-adjusted PSA levels between diabetics and non-diabetics only in European Americans (0.62 vs. $1.28 \mathrm{ng} / \mathrm{mL} ; \mathrm{p}=0.003$ ) and Latinos (0.99 vs. $1.27 \mathrm{ng} / \mathrm{mL}$; $\mathrm{p}=0.02$ ) but not in African Americans, Hawaiians and Japanese Americans.

In addition, in two studies, BMI did not confound the relationship between PSA and diabetics/non-diabetics $[26,28]$. When stratified by age $[18,25]$, the studies showed the lowest mean PSA for the youngest age group in both diabetics or non-diabetics. In one of the studies [18], diabetics showed a statistically significant reduction in PSA levels compared to non-diabetics for the age interval from 50 to 59 years mean (sd) PSA $1.03(1.09) \mathrm{ng} / \mathrm{mL}$ and $1.16(0.74) \mathrm{ng} / \mathrm{mL}$, respectively (p value: 0.0004$)$. The other study [25] obtained statistically significant differences for each age range (PSA levels were lower in diabetics than in non-diabetics) except for the age range 40 to 49 years.

A Chinese study [21] showed significantly higher PSA values (as mean of square root transformation PSA) in prediabetic subjects $(1.429 \mathrm{ng} / \mathrm{mL}$, sd: 0.353$)$ compared to normoglycaemic subjects $(1.086 \mathrm{ng} / \mathrm{mL}$, sd: 0.435$)$ and type 2 diabetes $(1.071 \mathrm{ng} / \mathrm{mL}$, sd: 0.420$)$ in agematched analysis but showed no difference between 
normoglycaemic and diabetic subjects. PSA level was significantly correlated with diabetes $(r=-0.203$, $\mathrm{p}=0.006 ; \mathrm{b}=-0.206, \mathrm{p}=0.005)$ and prediabetes $(\mathrm{r}=0.400$, $\mathrm{p}<0.001 ; \mathrm{b}=0.392, \mathrm{p}<0.001)$, both statistical models included age, BMI, Blood pressure, fasting plasma glucose, total cholesterol, triglycerides, HDL cholesterol, metabolic syndrome and T2DM or prediabetes, respectively [21]. In addition, a longitudinal study [28] presented results which suggest that Caucasian men with type 2 diabetes have smaller annual increases in serum PSA levels as they age than men without diabetes.

Only three studies explored the relationship between PSA and the duration of diabetes. Serum PSA level in diabetic patients was inversely influenced by duration of the disease $[19,26]$. PSA levels were lowest in men diagnosed with diabetes more than 10 years previously [26]. However, another study did not find an association between PSA and the duration of diabetes [24].

\section{Meta-analysis}

The PSA levels in diabetics and non-diabetics from the 8 studies included in the meta-analysis [18-22, 24, 25, 27] are shown in Table 2. In diabetics, mean (sd) PSA was 1.10 (0.38) ng/mL and $1.17(0.56) \mathrm{ng} / \mathrm{mL}$ in non-diabetics. There was significant heterogeneity in the included studies $\left(I^{2}=61.1 \%, p=0.012\right)$. This was accounted for using randomeffects meta-analyses. Overall, our analysis of the eight included studies revealed significant differences in the PSA level in diabetics compared with non-diabetics $(-0.07 \mathrm{ng} /$ $\mathrm{mL} ; 95 \%$ CI: $-0.10,-0.04$, Figure 2). In age-stratified analysis, significant differences in PSA levels between diabetics and non-diabetics were observed only for those aged 60 years and older $(-0.18$; 95\% CI: $-0.27,-0.09)$ compared to those under $60(-0.05 ; 95 \% \mathrm{CI}:-0.12,0.01)$ (Figure 3).

Heterogeneity is considerably reduced when we exclude the article Al-Asadi et al. [19] $\left(\mathrm{I}^{2}<50 \%\right)$ and Q statistic value was $>0.10$ (Table 3 ). In addition, the meta-regression showed significant influence in the association between diabetes and PSA according to the type of clinical population used for the study ( $\beta=-0.14,95 \%$ IC: $-0.24,-0.04 ; \mathrm{p}=0.006$ ). Studies carried out within the setting of general health screening showed lower differences in mean PSA levels between diabetics and non-diabetics compared to other settings such as population cohort. However, the metaregression showed no significant influence from study quality, the proportion of diabetics included in the study or significant differences in mean age or BMI between diabetics and non-diabetics. The Begg test suggested that that there was no significant publication bias in our meta-analysis ( $\mathrm{p}=0.06)$.

\section{Influence of diabetes treatment on PSA levels}

Out of the eight studies that assessed the effect of diabetes treatment on PSA levels, 5 (63\%) showed significantly lower PSA levels in diabetic men taking diabetes medication than in those without treatment [20,29] or compared to non-diabetic [24, 26, 27]. If the treatment included insulin, PSA levels were significantly lower than those taking only oral anti-diabetics [19]. However, other studies did not show significant differences [22, 26, 30] (see Supplementary Table $\mathrm{S} 3$ for additional details).

The studies included oral anti-diabetics such as metformin [20,29], with reported PSA values 30\% lower among metformin users [29]. A study included the treatment with thiazolidinedione without significant differences in PSA levels [30]. In addition, insulin treatment also showed lower PSA levels compared with those not treated with insulin [19], and even lower PSA levels compared to patients treated with oral anti-diabetics [24]. Duration of metformin use did not appear to influence PSA levels [20] but metformin dose dependency was inversely associated with PSA levels [29].

\section{Quality assessment}

The results of the quality assessment for the studies included in the meta-analysis are summarized in Table 2. The quality assessment of the complete set of 13 studies included in the systematic review can found in the Supplementary Table S4 and include more detail for each category assessed. Among these 11 studies that assessed the influence of diabetes on PSA level, 3 (27.3\%) were considered to be of strong quality, 1 (9.1\%) weak quality and the rest $(6,54.6 \%)$ showed moderate quality. Selection bias was considered as possible in $8(72.7 \%)$, the methods used to ascertain information on the exposure (diabetes) were classed as unclear in $6(54.6 \%)$ studies and one of the studies (9.1\%) had not considered important confounding variables in the analysis.

Among the studies that assessed the effect of diabetes treatment on PSA levels 7 (87.5\%) presented moderate quality and $1(12.5 \%)$ strong quality. Selection bias was considered as possible in $6(75.0 \%)$ studies, and the methods for ascertaining information about the exposure (diabetes treatment) was questionable in four studies (patient self-report). 


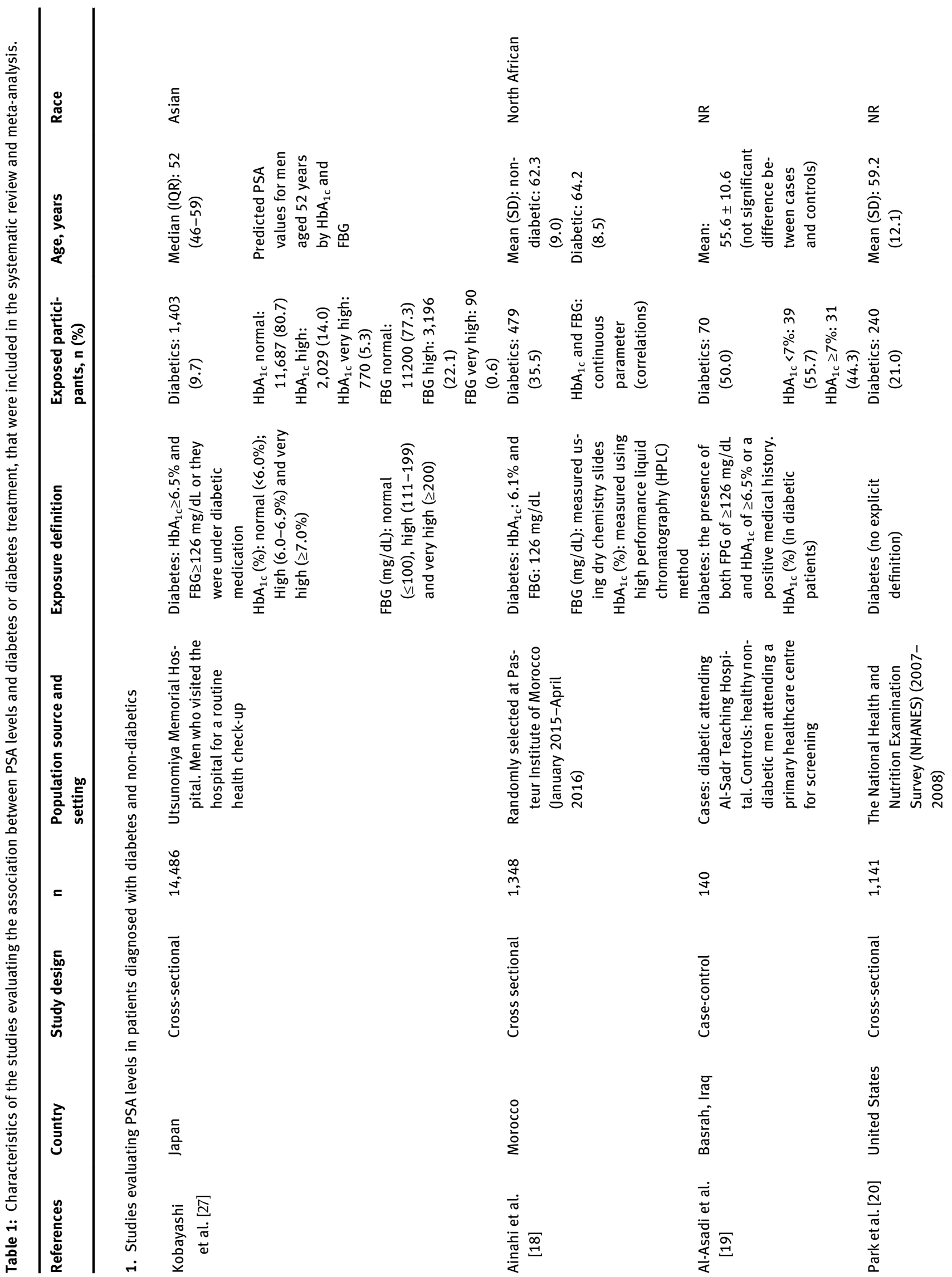




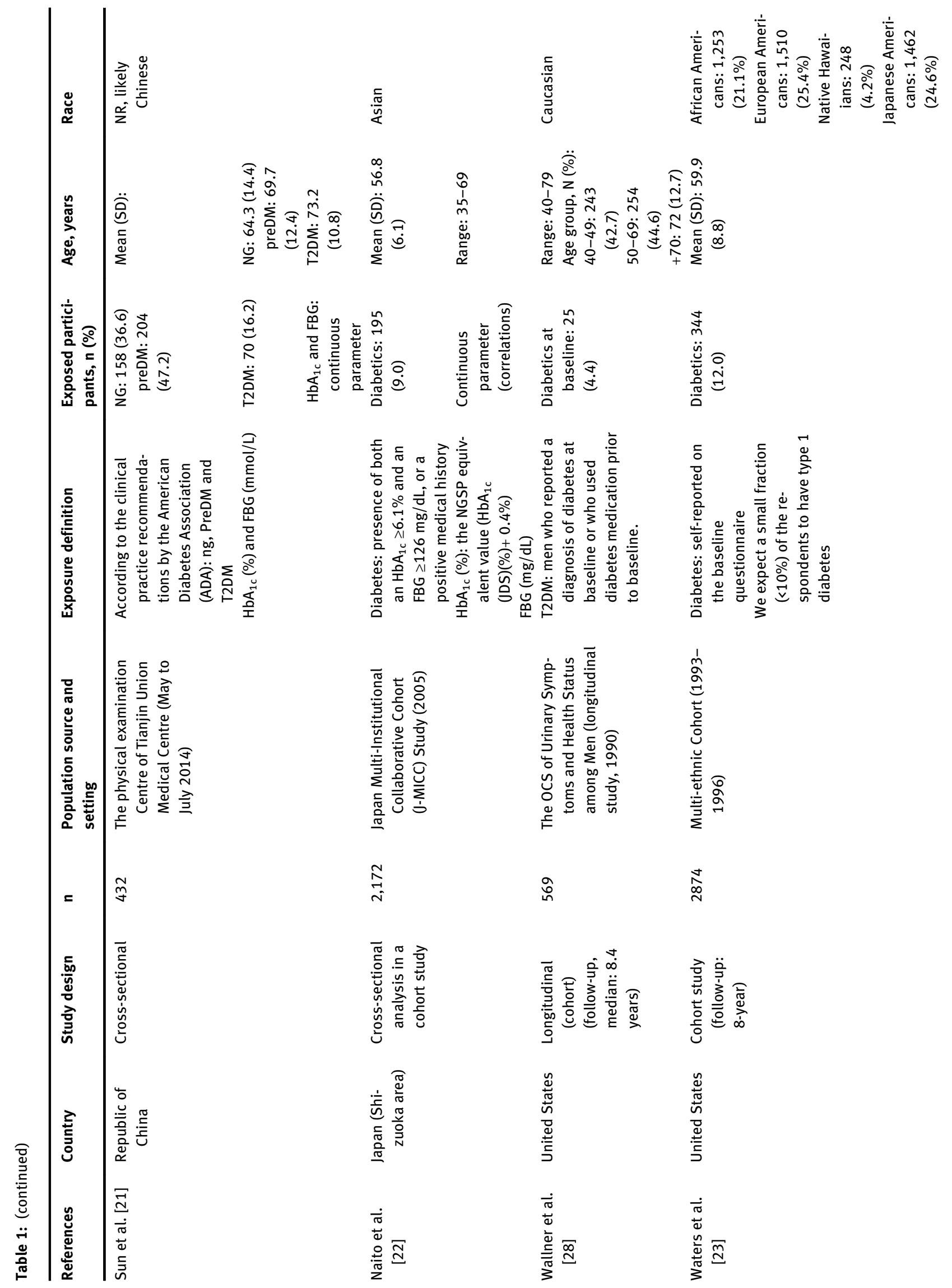




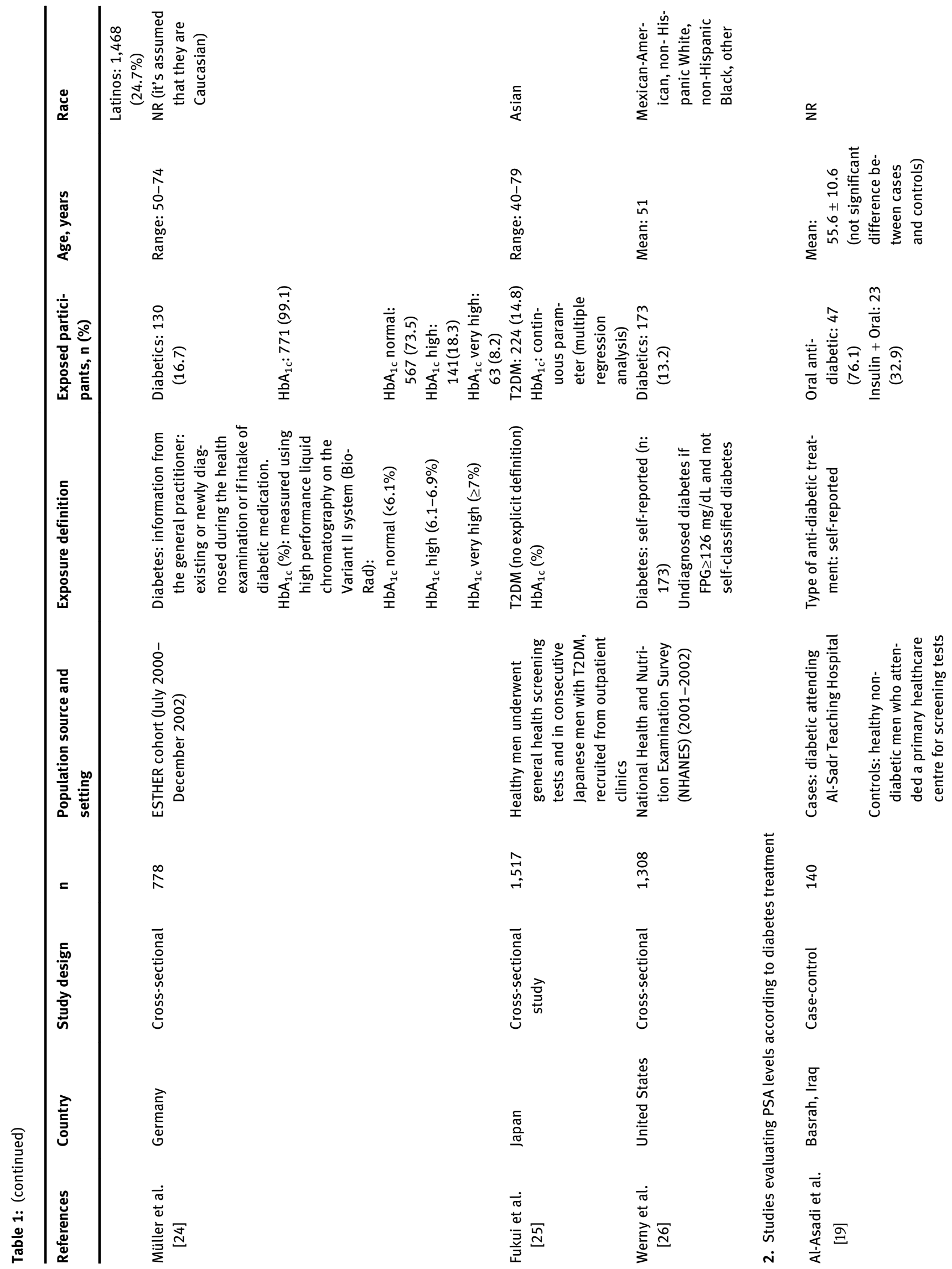




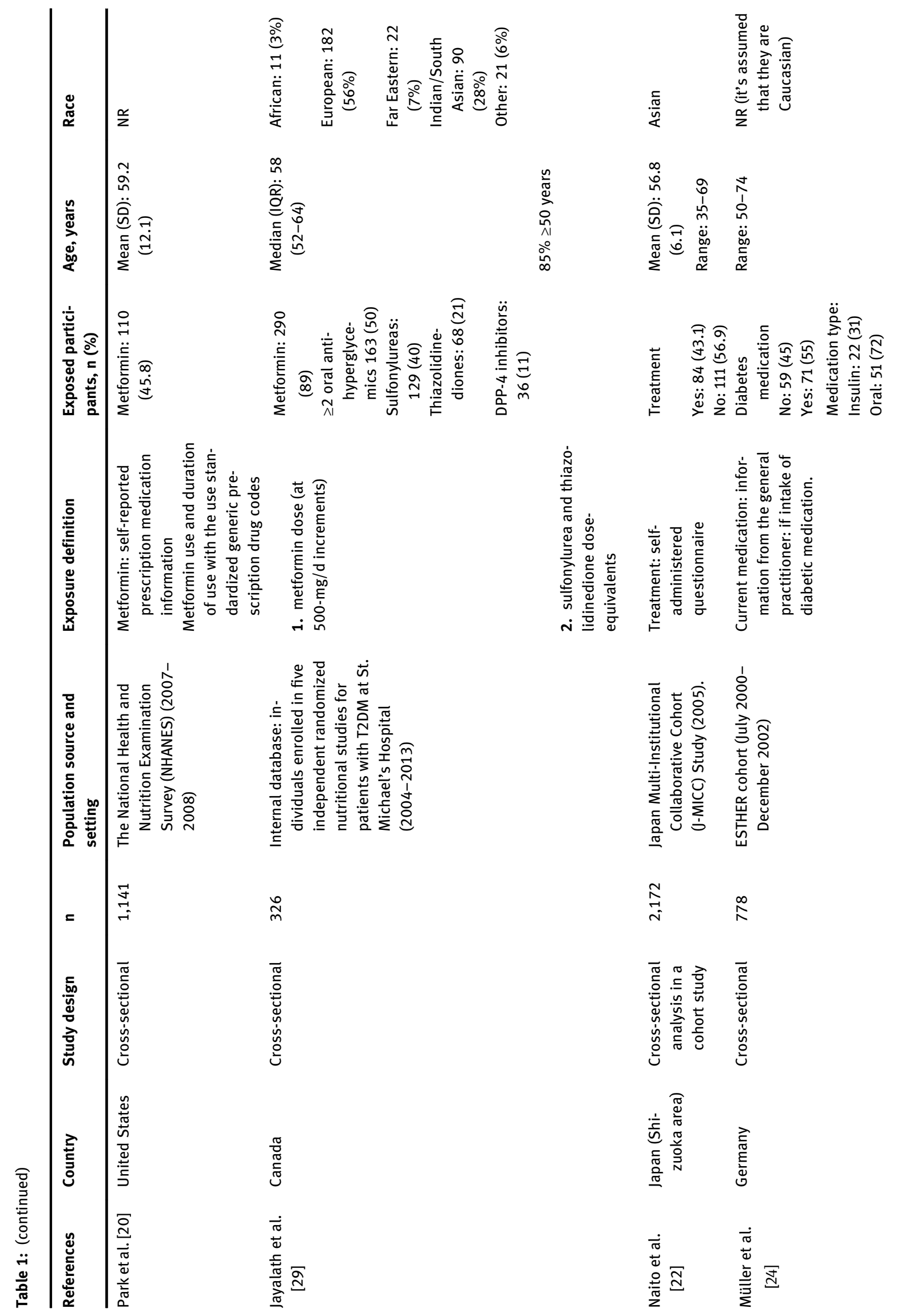




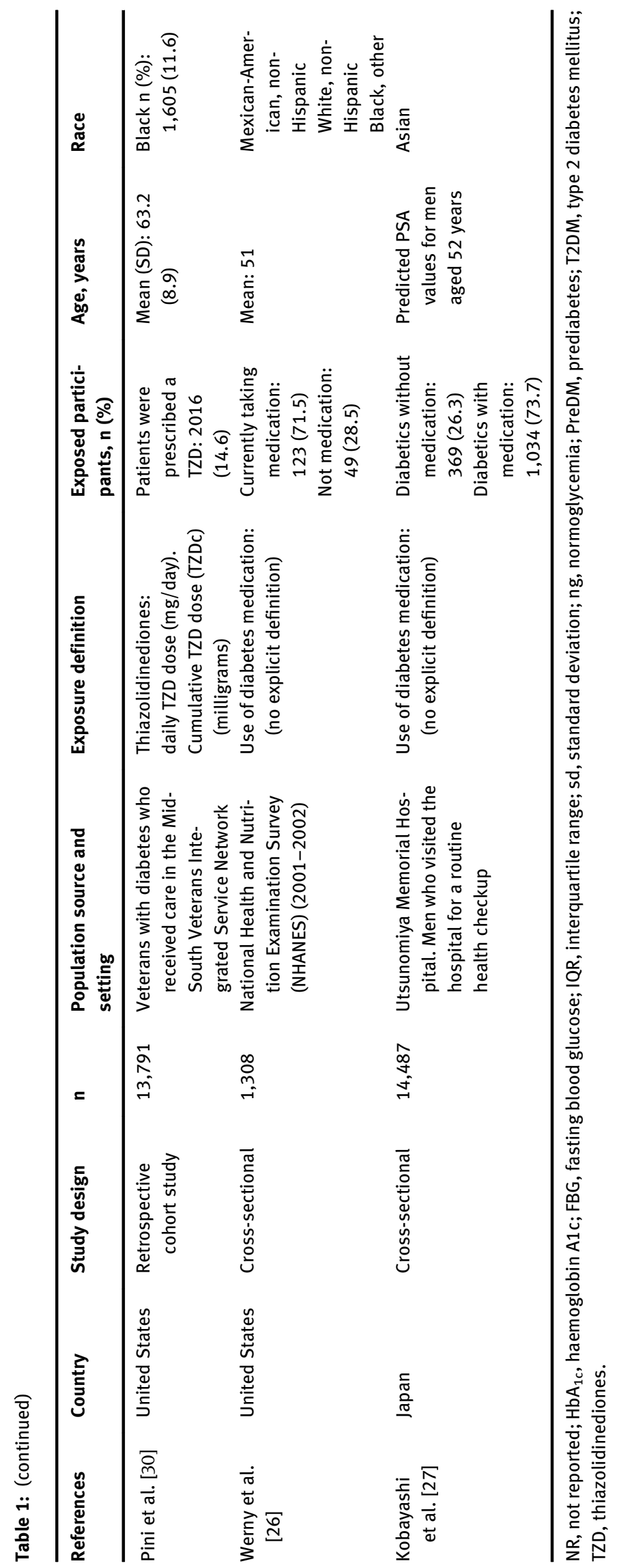




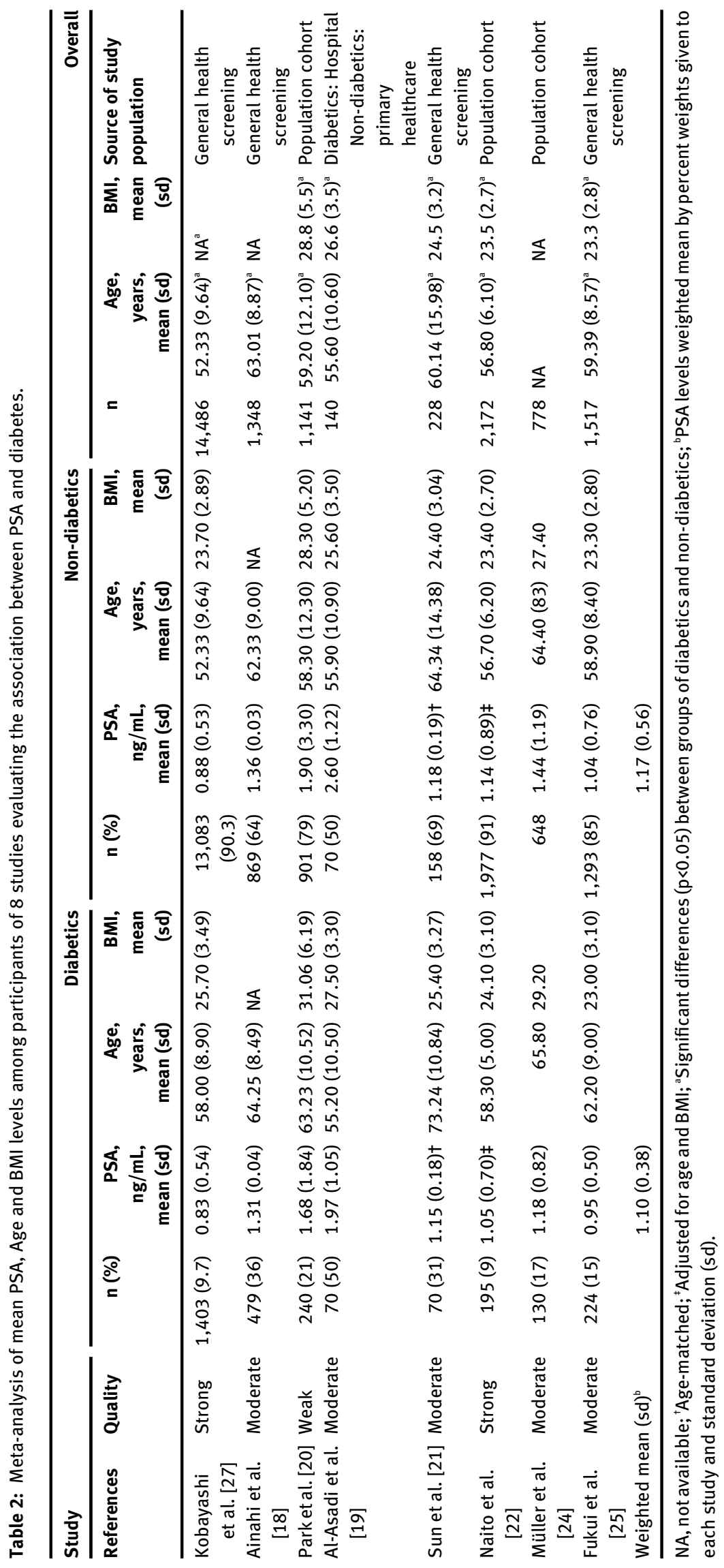




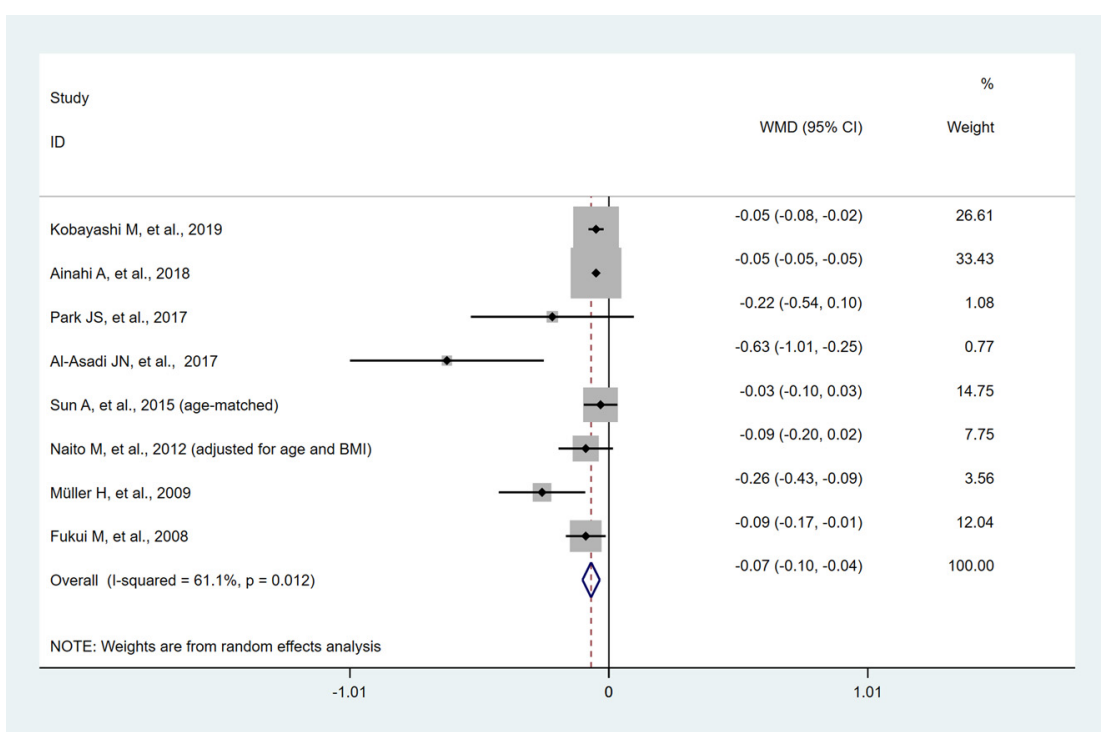

Figure 2: Forrest plot of eight studies analyzing the mean difference in PSA levels $(\mathrm{ng} / \mathrm{mL})$ between diabetic and non-diabetic men.
Figure 3: Forrest plot of three studies analyzing the age-stratified mean difference in PSA levels $(\mathrm{ng} / \mathrm{mL})$ between diabetic and non-diabetic men.

Table 3: Sensitivity analysis.

\begin{tabular}{lrrrrrr}
\hline Study omitted & Mean difference & & $\mathbf{9 5 \%} \mathbf{C l}$ & Mean difference change, \% & $\mathbf{I}^{2}$, (\%) & Homogeneity Chi-square (p) \\
\hline Fukui (2008) & -0.050 & -0.054 & -0.046 & -0.22 & 64.7 & 0.009 \\
Müller (2009) & -0.050 & -0.054 & -0.046 & -0.24 & 50.1 & 0.061 \\
Naito (2012) & -0.050 & -0.054 & -0.046 & -0.12 & 65.7 & 0.008 \\
Sun (2015) & -0.050 & -0.054 & -0.046 & 0.14 & 66.1 & 0.007 \\
Al-Asadi (2017) & $-\mathbf{0 . 0 5 0}$ & $-\mathbf{0 . 0 5 4}$ & $-\mathbf{0 . 0 4 6}$ & $-\mathbf{0 . 1 3}$ & $\mathbf{3 2 . 8}$ & $\mathbf{0 . 1 7 7}$ \\
Park (2017) & -0.050 & -0.054 & -0.046 & -0.05 & 64.5 & 0.010 \\
Ainahi (2018) & -0.062 & -0.086 & -0.037 & 22.30 & 65.1 & 0.009 \\
Kobay (2019) & -0.050 & -0.054 & -0.046 & 0.01 & 66.7 & $\mathbf{6 1 . 1}$ \\
None & $-\mathbf{0 . 0 5 0}$ & $-\mathbf{0 . 0 5 4}$ & $-\mathbf{0 . 0 4 6}$ & $\mathbf{0}$ & $\mathbf{6 1 . 1}$ & $\mathbf{0 . 0 1 2}$ \\
\hline
\end{tabular}

Bold values denote results without excluding any study from analysis and results that do not show significant heterogeneity. 


\section{Discussion}

Most of the studies included showed significant differences in PSA levels between diabetics and non-diabetics when controlling by age [18, 22-28] and race [23]. However, there were contradictory results when controlling by BMI [19, 21, 22, 24, 26]. The meta-analysis results showed that diabetic patients had a significantly lower PSA level compared to non-diabetic patients. Nevertheless, the mean difference value obtained was low $(-0.07,95 \% \mathrm{CI}:-0.10,-0.04)$, with values from -0.03 [21] to -0.63 [19] in the individual studies.

The level of PSA in the blood increases with age by about 3.2\% per year [31]. It has been suggested that the sensitivity of the PSA test for detecting PCa could be improved by applying age-specific reference ranges. Therefore, it is an essential confounding factor to control for when we analyse the different PSA levels between diabetic and non-diabetic patients. In this review, PSA levels were found to decrease in diabetic patients in comparison with nondiabetic patients when controlling by age.

Our age-stratified analysis meta-analysis showed that the difference in PSA levels between diabetics and nondiabetics was only significant for those over 60. It is possible that differences in population characteristics may have contributed to this low absolute difference, especially if there were large differences in age. Although most of the individual studies showed a significant difference when controlling by age or BMI, we collected data from published articles and adjusted values for these parameters were only available for some of them. Race may influence in the relationship between PSA levels and diabetes [23]. For example, studies carried out in Asian population $[21,22,25]$ characterized by low obesity prevalence, which is a possible confounding factor [32,33], showed lower PSA levels in diabetics in comparison with diabetics.

Some studies have not included data on diabetes mellitus type $[18,20,22-24,26]$, severity $[18,20-23,28]$ or duration $[18,20-23,25,28]$ which are essential factors to consider in the analysis. Some articles for instance, showed that serum PSA level in diabetic patients was inversely influenced by duration of the disease [19, 26]. This fact may be explained by the low level of IGF-1 in long-term diabetes [34] as insulin production drops [35] may further explain the low level of PSA in diabetic patients [19]. However, in contrast with previous studies [36], we found contradictory results in the association between $\mathrm{HbA}_{1 \mathrm{c}}$ and PSA levels [18, $19,22,24,25,27,37,38$ ], perhaps due to the lack of other controlling data in these studies such as duration of the disease, BMI or total testosterone concentration.
Half of the studies showed significantly lower PSA levels in diabetic men taking either oral diabetes medication [20, 24, 26, 29] or insulin [19]. According to our results, patients with anti-diabetic medications particularly metformin [20] may have reduced serum total prostate-specific antigen [29]. These findings are in line with a recent publication [39] which found that the association between the use of metformin and a low risk of PCa, especially of localized disease in men with a long history of diabetes, may be explained by detection bias due to lower PSA levels in this group. However, they also indicated that it is difficult to distinguish the specific anti-diabetic drug effect from that of underlying diabetes. In our systematic review, patients with anti-diabetic treatment showed lower PSA levels than untreated diabetics [24, 26, 27].

In addition, diabetic men on insulin combined with oral treatment had lower serum PSA level than those on oral anti-diabetic medications alone [19]. Although unlike metformin use, insulin is not associated with a lower risk of $\mathrm{PCa}$ [39]. Use of insulin may be an approximate surrogate of diabetes severity [24] and an indicator of a later stage of diabetes that is characterised by a lower level of circulating insulin and insulin resistance and consequently low serum PSA concentration [28]. This is in agreement with the fact that serum PSA concentration significantly decreased with increasing quartile of insulin resistance [40, 41].

There are several possible explanations for PSA being lower in diabetic men than in those non-diabetic men, including greater obesity, more frequent use of medications to treat dyslipidemia, microvascular complications, which contribute to prostate ischemia, and lower serum androgen levels. In addition, since PSA is androgen regulated [42] such association may be partly explained by lower serum testosterone concentration among diabetic patients [43].

Although, previous studies that evaluated the relationship between PSA and diabetes status showed contradictory results, our results indicate that interpretation of PSA levels may need to change in accordance with diabetes status. However, the consideration is not a simple yes-no question. It appears that other factors such as the duration $[19,26]$ or control of the disease according to $\mathrm{HbA}_{1 \mathrm{c}}$ levels $[19,24,37]$ or diabetes treatments $[19,24,29]$ may influence PSA levels and not the fact of having a diabetes diagnosis. According to previous evidence, an age-BMI-adjusted PSA model is no more clinically useful for detecting prostate cancer than current National Institute for Health and Care Excellence (NICE) guidelines which advise using an agespecific cut-off [44]. The usefulness of establishing ageand diabetes-specific cut-offs should also be assessed. 
A recently published systematic review [39] indicated that the potential inverse association between diagnosis of diabetes and PCa among the PSA-screened population seems to be restricted to total and low-grade/localized disease. Given that mean PSA values were significantly lower for men with diabetes, the reduced risk of lowmoderate-grade but not of high-grade PCa among men with diabetes may suggest a potential detection bias.

The small differences observed in mean PSA values among diabetics and non-diabetics could have little practical value if we consider the potential for analytical variability in the studies observed. Although the majority of studies indicated that all samples from diabetic and non-diabetic patients were analysed with the same analyser, not all studies used the same analyser. A variability higher than $10 \%$ between different commercial assays has been associated with a significant impact on the clinical classification of patients, corresponding to an overall false recommendation rate of $2.5 \%$ including false-positive and false-negative results [45]. Furthermore, significant analytical variation has been observed using different reagents on the same analyser (ARCHITECT i2000) [46], and the potential for between-subject biological variation in PSA levels in healthy subjects is not clear [47]. That being said, differences in PSA levels between diabetics and non-diabetics were observed in all studies and only in three studies were they not statistically significant.

There are several limitations in our study. Firstly, our search was limited to research published in indexed journals. Moreover, the power of analysis examining the association between DM and PSA levels was limited by the reduced number of included studies, although most of them had a considerable sample size. In four studies [22, $24,25,27]$ the proportion of diabetics was less than $20 \%$ which could decrease the statistical power to analyze the impact of diabetes on PSA levels in these studies. In addition, we are unable to assess the association between PSA levels and type 1 or type 2 DM separately. Another limitation of the included studies is that they did not consider prostate volume as a potentially confounding factor. This could be relevant given that diabetes has been associated with larger prostate sizes [48]. In our quality analysis we noted some significant methodological deficits, especially in the studies that assessed the influence of diabetes treatment on PSA levels. Although it would be preferable to use a single tool for analyzing study quality, the included studies included a mix of designs and methodologies and we felt it was more appropriate to apply selected risk of bias questions from three different guidelines. Lastly, heterogeneity related to differences across studies in patient selection, adjustment for potential confounders, and information on DM duration could influence the results. These factors make interpreting the results of the meta-analysis more challenging and the decision to carry out a formal meta-analysis was not straight forward. We carried out a sensitivity analysis and a meta-regression assess the impact of the heterogeneity. There was a significant difference in PSA levels between diabetics and non-diabetics independently of the inclusion or exclusion of the study responsible for heterogeneity.

\section{Conclusions}

Diabetic men showed lower PSA levels than non-diabetic men. Lower PSA levels in diabetics seem to be related with factors like diabetes duration, severity of the disease, or taking diabetes medication rather than the actual diagnosis of diabetes. The differences were very small and although they seem to be greater with increasing age, it is unlikely that using a diabetes specific cut-off will improve accuracy of PSA screening.

Research funding: This study was funded by the Institute of Health Carlos III (Ministry of Economy and Competitiveness, MINECO), and by the European Regional Development Fund (ERDF) “A Way to Make Europe” Grant No.: PI17/01883.

Author contributions: All authors have accepted responsibility for the entire content of this manuscript and approved its submission.

Competing interests: Authors state no conflict of interest.

\section{References}

1. American Urological Association. Available at: https://www. auanet.org/guidelines/prostate-cancer-early-detection(2013-reviewed-for-currency-2018) [Accessed 25 Jan 2019].

2. European Association of Urology. Available at: https://uroweb. org/guideline/prostate-cancer/ [Accessed 25 Jan 2019].

3. Mottet N, Bellmunt J, Bolla M, Briers E, Cumberbatch MG, De Santis $M$, et al. EAU-ESTRO-SIOG guidelines on prostate cancer. part 1: screening, diagnosis, and local treatment with curative intent. Eur Urol 2017;71:618-29.

4. Grossman DC, Curry SJ, Owens DK, Bibbins-Domingo K, Caughey $A B$, Davidson KW, et al. Screening for prostate cancer. JAMA. 2018; 319:1901.

5. Shakir NA, George AK, Siddiqui MM, Rothwax JT, Rais-Bahrami S, Stamatakis $L$, et al. Identification of threshold prostate specific antigen levels to optimize the detection of clinically significant prostate cancer by magnetic resonance imaging/ultrasound fusion guided biopsy. J Urol 2014;192:1648.

6. Schröder FH, Roobol MJ. Defining the optimal prostate-specific antigen threshold for the diagnosis of prostate cancer. Curr Opin Urol 2009;19:227-31.

7. Hatakeyama S, Yoneyama T, Tobisawa Y, Ohyama C. Recent progress and perspectives on prostate cancer biomarkers. Int J Clin Oncol 2017;22:214-21. 
8. De Nunzio C, Tubaro A. Diabetes and prostate cancer-an open debate. Nat Rev Urol 2013;10:12-4.

9. Pierce BL. Why are diabetics at reduced risk for prostate cancer? A review of the epidemiologic evidence. Urol Oncol Semin Orig Invest 2012;30:735-43.

10. Dhindsa SS, Irwig MS, Wyne K. Gonadopenia and aging in men. Endocr Pract 2018;24:375-85.

11. Moher D, Liberati A, Tetzlaff J, Altman DG. Preferred reporting items for systematic reviews and meta-analyses: the PRISMA statement. PLoS Med 2009;6:e1000097.

12. Wells GA, Shea B, O'Connell D, Peterson J., Welch V, Losos M, et al. The Newcastle-Ottawa Scale (NOS) for assessing the quality of nonrandomised studies in meta-analyses; 2011. Available at: http://www.ohri.ca/programs/clinical_epidemiology/oxford. asp [Accessed 3 Jan 2019].

13. Sterne J, Higgins J, Reeves B, ACROBAT-NRSI on behalf of the development group for. A Cochrane Risk of Bias Assessment Tool: for Non-Randomized Studies of Interventions (ACROBAT-NRSI), Version 1.0.0; 2014. Available at: http://www.riskofbias.info [Accessed 3 Jan 2019].

14. National Collaborating Centre for Methods and Tool. Quality assessment tool for quantitative studies. Hamilton, Canada; 2008. Available at: https://www.nccmt.ca/knowledgerepositories/search/14 [Accessed 3 Jan 2019].

15. Luo D, Wan X, Liu J, Tong T. Optimally estimating the sample mean from the sample size, median, mid-range, and/or mid-quartile range. Stat Methods Med Res 2018;27:1785-805.

16. Wan X, Wang W, Liu J, Tong T. Estimating the sample mean and standard deviation from the sample size, median, range and/or interquartile range. BMC Med Res Methodol 2014;14:135.

17. Higgins JP, Green S. Selecting studies and collecting data. In: Higgins JPT, Deeks JJ, editors. Cochrane handbook for systematic reviews of interventions. Chichester, UK: John Wiley \& Sons; 2008, pp. 151-83.

18. Ainahi A, Barakat A, Wakrim L, Mohammadi H, El-Mdaghri N, Ezzikouri S. Prostate-specific antigen levels in moroccan diabetic males: a cross-sectional study. Curr Diabetes Rev 2018;14: 286-90.

19. Al-Asadi J, Al-Naama L, Abdul-Kareem M, Mashkoor F. Serum level of prostate-specific antigen in diabetic patients in Basrah, Iraq. Niger Postgrad Med J 2017;24:240.

20. Park JS, Lee KS, Ham WS, Chung BH, Koo KC. Impact of metformin on serum prostate-specific antigen levels. Medicine (Baltimore) 2017;96:e9427.

21. Sun A, Liu R, Sun G. Serum prostate-specific antigen levels in men with prediabetes: A cross-sectional study. Scand J Clin Lab Invest 2015;75:273-81.

22. Naito M, Asai Y, Mori A, Fukada Y, Kuwabara M, Katase S, et al. Association of obesity and diabetes with serum prostate-specific antigen levels in Japanese males. Nagoya J Med Sci 2012;74: 285-92.

23. Waters KM, Henderson BE, Stram DO, Wan P, Kolonel LN, Haiman CA. Association of diabetes with prostate cancer risk in the multiethnic cohort. Am J Epidemiol 2009;169: 937-45.

24. Muller H, Raum E, Rothenbacher D, Stegmaier C, Brenner H. Association of diabetes and body mass index with levels of prostate-specific antigen: implications for correction of prostatespecific antigen cutoff values? Cancer Epidemiol Biomarkers Prev 2009;18:1350-6.
25. Fukui M, Tanaka M, Kadono M, Imai S, Hasegawa G, Yoshikawa T, et al. Serum prostate-specific antigen levels in men with type 2 diabetes. Diabetes Care 2008;31:930-1.

26. Werny DM, Saraiya M, Gregg EW. Prostate-specific antigen values in diabetic and non-diabetic US men, 2001-2002. Am J Epidemiol 2006;164:978-83.

27. Kobayashi M, Mizuno T, Yuki H, Kambara T, Betsunoh H, Nukui A, et al. Association between serum prostate-specific antigen level and diabetes, obesity, hypertension, and the laboratory parameters related to glucose tolerance, hepatic function, and lipid profile: implications for modification of prostate-specific antigen threshold. Int J Clin Oncol 2020;25:472-8.

28. Wallner LP, Morgenstern H, McGree ME, Jacobson DJ, St Sauver JL, Jacobsen SJ, et al. The effects of type 2 diabetes and hypertension on changes in serum prostate specific antigen levels: results from the Olmsted County study. Urology 2011;77:137-41.

29. Jayalath VH, Ireland C, Fleshner NE, Hamilton RJ, Jenkins DJA. The relationship between metformin and serum prostate-specific antigen levels. Prostate 2016;76:1445-53.

30. Pini TM, Griffin MR, Roumie CL, Huizinga MM, Fowke JH, Greevy R, et al. Use of thiazolidinediones does not affect prostate-specific antigen levels in men with diabetes. Cancer Epidemiol Biomarkers Prev 2009;18:1937-8.

31. Oesterling JE, Jacobsen SJ, Chute CG, Guess HA, Girman CJ, Panser LA, et al. Serum prostate-specific antigen in a community-based population of healthy men: establishment of age-specific reference ranges. JAMA J Am Med Assoc 1993;270: 860-4.

32. Yang HJ, Doo SW, Yang WJ, Song YS. Which obesity index best correlates with prostate volume, prostate-specific antigen, and lower urinary tract symptoms? Urology 2012;80:187-90.

33. Litchfield MJ, Cumming RG, Smith DP, Naganathan V, Le Couteur DG, Waite LM, et al. Prostate-specific antigen levels in men aged 70 years and over: findings from the CHAMP study. Med J Aust 2012;196:395-8.

34. Teppala S, Shankar A. Association between serum IGF-1 and diabetes among U.S. adults. Diabetes Care 2010;330:22579.

35. Giovannucci E. Nutrition, insulin, insulin-like growth factors and cancer. Horm Metab Res 2003;35:694-704.

36. Atalay HA, Akarsu M, Canat L, Ülker V, Alkan I, Ozkuvancı U. Impact of poor glycemic control of type 2 diabetes mellitus on serum prostate-specific antigen concentrations in men. Prostate Int 2017;5:104-9.

37. Fowke JH, Matthews CM, Buchowski MS, Signorello LB, Chang SS, Cookson MS, et al. Association between prostate-specific antigen and leptin, adiponectin, $\mathrm{HbA}_{1 \mathrm{c}}$ or $\mathrm{C}$-peptide among African-American and Caucasian men. Prostate Cancer Prostatic Dis 2008;11:264-9.

38. Ohwaki K, Endo F, Muraishi O, Yano E. Relationship between changes in haemoglobin $A_{1 C}$ and prostate-specific antigen in healthy men. Eur J Cancer 2011;47:262-6.

39. Campi R, Brookman-May SD, Subiela Henríquez JD, Akdoğan B, Brausi $\mathrm{M}$, Klatte $\mathrm{T}$, et al. Impact of metabolic diseases, drugs, and dietary factors on prostate cancer risk, recurrence, and survival: a systematic review by the European association of urology section of oncological urology. Eur Urol Focus 2019;5: 1029-57.

40. Han JH, Lee YT, Kwak KW, Ahn SH, Chang IH, Myung SC, et al. Relationship between insulin resistance, obesity and serum 
prostate-specific antigen levels in healthy men. Asian J Androl 2010;12:400-4.

41. Choi H-C, Park J-H, Cho B-L, Son K-Y, Yoo Y-J, Kwon H-T. The illusion of prostate-specific antigen decline in patients with metabolic syndrome and insulin resistance. BJU Int 2011;108:1756-61.

42. Balk SP, Ko YJ, Bubley GJ. Biology of prostate-specific antigen. J Clin Oncol 2003;21:383-91.

43. Mattack N, Devi R, Kutum T, Patgiri D. The evaluation of serum levels of testosterone in type 2 diabetic men and its relation with lipid profile. J Clin Diagnostic Res 2015;9:BC04-7.

44. Harrison S, Tilling K, Turner EL, Lane JA, Simpkin A, Davis M, et al. Investigating the prostate specific antigen, body mass index and age relationship: is an age-BMI-adjusted PSA model clinically useful? Cancer Causes Control 2016;27:1465-74.

45. Foj L, Filella X, Alcover J, Augé JM, Escudero JM, Molina R. Variability of assay methods for total and free PSA after WHO standardization. Tumor Biol 2014;35:1867-73.
46. Kittanakom S, Clark L, Roy C, Malinowski P, Bamford K, Lamers S, et al. Differences in total PSA results within and between manufacturers. Clin Biochem 2018;60:91-2.

47. Carobene A, Guerra E, Locatelli M, Cucchiara V, Briganti A, Aarsand AK, et al. Biological variation estimates for prostate specific antigen from the European Biological Variation Study; consequences for diagnosis and monitoring of prostate cancer. Clin Chim Acta 2018;486:185-91.

48. Mubenga LE, Hermans MP, Chimanuka D, Muhindo L, Cikomola J, Bahizire E, et al. Anthropometric and cardiometabolic correlates of prostate volume among diabetic and non-diabetic subjects in South-Kivu. Diabetes Metab Syndr Clin Res Rev 2019;13:350-5.

Supplementary Material: The online version of this article offers supplementary material https://doi.org/10.1515/cclm-2020-0145. 